\title{
Design and Development of a Novel Controller for Robust Stabilisation and Attitude Control of an Unmanned Air Vehicle for Nuclear Environments*
}

\author{
Hamidreza Nemati \\ Engineering Department \\ Lancaster University \\ Lancaster LA14YW, UK \\ h.nemati@lancaster.ac.uk
}

\author{
Allahyar Montazeri \\ Engineering Department \\ Lancaster University \\ Lancaster LA14YW, UK \\ a.montazeri@lancaster.ac.uk
}

\begin{abstract}
This study presents two new robust nonlinear control algorithms based on the theory of time-varying sliding mode control (TVSMC) strategy to stabilize the attitude of an unmanned aerial vehicle (UAV) for nuclear decommissioning applications. Constant radiation affects the UAV performance. For instance, its parameters are time-varying and subject to uncertainty all the time. This is especially important in designing sliding mode control as the motion of the control system in the reaching phase is highly sensitive against environmental disturbances and parameter uncertainties. In this study, two types of time-varying sliding manifolds are proposed to eliminate the reaching phase and to enhance the robust performance in the aforementioned phase. Therefore, two novel types of time-varying sliding surfaces are introduced based on the initial condition as intercept-varying sliding mode control (IVSMC) approaches. In the first proposed method, the reaching time from initial manifold to the desired one is similar to that of the conventional SMC method. While in the second proposed IVSMC scheme, one can accelerate or decelerate the motion of the time-varying sliding manifolds at any selected time. Furthermore, chattering phenomenon can be avoided using two techniques known as boundary layer and continuous SMC. Finally, to highlight the robust performance of the proposed methods, a quadrotor UAV subject to external disturbances is simulated.
\end{abstract}

Index Terms-Sliding mode control, Intercept-varying, Timevarying manifolds, Reaching time, Lyapunov stability, Boundary layer, Continuous SMC, Quadrotor UAV

\section{INTRODUCTION}

As many nuclear power plants and facilities in UK and around the world are reaching to the end of their operating life, they have imposed a great challenge for decommissioning and demolishing process. This is partly due to imposing significant risks to the people and the environment and is partly related to the restrictions imposed for human access due to highradiation level of the facilities and infrastructures. Although remote and robotic decommissioning solutions provide a way forward, there are still challenging problems to be solved [1]. The use of hydraulic manipulators has been proven an effective solution for decommissioning in nuclear industries

The work is supported by the Engineering and Physical Sciences Research Council (EPSRC), grant number EP/R02572X/1, and National Centre for Nuclear Robotics
[2], [3]. Due to unknown and time-varying parameters of such complex electro-mechanical systems nonlinear optimisation techniques such as those applied in [4] and [5] are required to develop a reliable model. Nevertheless, due to large sizes of manipulators, other robotic platforms such as Unmanned Aerial Vehicles (UAVs) have also been deployed and tested for radiation mapping in decommissioning applications. Due to the flexibility to inspect and characterise a large area of environment UAVs are promising autonomous systems in decommission applications [1]. However, as nuclear plants may have complex structure of pipework, autonomous operation of the UAVs requires a high level of maneuverability. The problem gets more challenging since the guidance and navigation sensors are subject to constant radiations and hence are degraded by time. This necessitates development of a nonlinear control system which is robust against parameter uncertainties and unpredictable failure of sensors and actuators. Quadrotor UAVs have attracted much interest recently compared with unmanned fixed-wing flight vehicles due to many significant advantages such as stable hovering, vertical take-off and landing, small size, low cost and simple portability.

Sliding mode control (SMC) technique is one of the most promising robust control methodologies because of low sensitivity to plant parameter variations and disturbances which can diminish the burden of the necessity of exact modeling. The main attractive feature of SMC is to persuade the motion of the sliding mode toward the predetermined switching manifold. Basically, conventional SMC strategies include two phases: reaching phase and sliding phase. In the reaching phase, system's trajectory starts from a given initial condition, moves toward a predetermined sliding surface and reach it. In the sliding phase, the motion is restricted to the sliding manifold. Recently many studies and projects have been dedicated to the application of sliding mode controllers for quadrotor stabilization. In [6], position and attitude tracking problem for a quadrotor UAV have been introduced based on the SMC strategy subject to external disturbances. The limitation of robustness of the conventional sliding mode controller has been investigated in [7]. A novel robust generalized dynamic 
inversion control system to stabilize the position and attitude of a quadrotor has been presented in [8]. In this paper, the robustness issue is ensured by SMC method. In [9], a control methodology has been suggested for the quadrotor trajectory tracking problem using both integral backstepping and sliding mode controllers under external disturbances. Since the conventional backstepping controller can only deal with constant uncertainties, the combination of SMC with backstepping method has been considered.

To enhance the robust performance in SMC, Integral Sliding Mode Control (ISMC) was first elaborated in [10]. ISMC can eliminate the reaching phase and promote the robustness of the system starting from the initial time instance. In [11], the general case of both, matched and mismatched disturbances based on ISMC was addressed. To further improve the robust performance, double integral SMC (DISMC) has been utilized in [12], [13]. However, these methods have the following disadvantages:

- The order of the equation of motion in ISMC is of full order.

- The overcompensation of the integral term to big errors can deteriorate the transient response performance of the system which leads to a long convergence time.

Time-varying sliding mode control (TVSMC) has been proposed in [14]. Since the motion of the control system in the reaching phase is highly sensitive against environmental disturbances and parameter uncertainties, TVSMC is able to eliminate the reaching phase using time-varying sliding manifolds. However, the former method could not guarantee insensitivity of the system subject to uncertainties and disturbances for some time. Then, a continuous time-varying sliding manifold has been developed in [15]. TVSMC has proven to be successful in countless applications [16]. However, in these studies, the finite reaching time to the sliding manifold cannot be assured and the switching time must be designed using some complicated optimization approaches.

In this paper, two new continuous intercept-varying sliding mode control (IVSMC) approaches are introduced via shifting sliding manifolds. In the first approach, the time required for shifting from the initial surface to the final one is similar to the reaching time in the conventional SMC technique. In the second approach, one can easily accelerate or decelerate the reaching phase by selecting the optional time. One of the main contributions of the proposed IVSMC methods is to be available for every initial conditions in the phase plane. In addition, to eliminate the chattering drawback two techniques are employed using boundary layer and continuous SMC [17]. The remainder of the paper is organized as follows: Next section includes preliminaries for deriving an attitude model of the quadrotor UAV. Then, detailed theoretical analyses of the proposed SMC techniques are presented and compared against the conventional SMC. Next, the application of the proposed methods for UAVs using numerical simulations and analyses are given. Finally, the paper is ended with some concluding remarks.
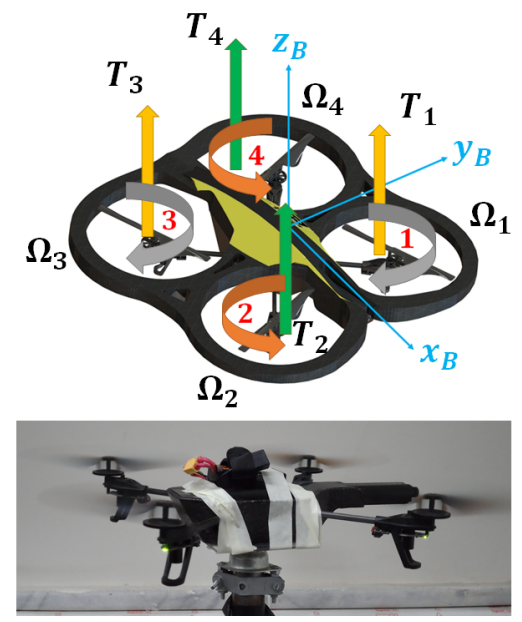

Fig. 1. Quadrotor model with coordinate axes

\section{Attitude Dynamics Description}

A quadrotor UAV consists of four rotors with bolted propellers in cross-shaped frame as depicted in Fig. 1. The attitude change of the quadrotor results from variations on forces and moments produced by adjusting rotors' speeds. The quadrotor attitude dynamics can be written in the following form [18]:

$$
\left[\begin{array}{c}
\ddot{\phi} \\
\ddot{\theta} \\
\ddot{\psi}
\end{array}\right]=\left[\begin{array}{c}
\frac{J_{y y}-J_{z z}}{J_{x x}} \dot{\theta} \dot{\psi} \\
\frac{J_{z z}-J_{x x}}{J_{y y}} \dot{\phi} \dot{\psi} \\
\frac{J_{x x}-J_{y y}}{J_{z z}} \dot{\phi} \dot{\theta}
\end{array}\right]-J_{r} \Omega_{r}\left[\begin{array}{c}
\frac{\dot{\theta}}{J_{x x}} \\
-\frac{\dot{\phi}}{J_{y y}} \\
0
\end{array}\right]+\left[\begin{array}{c}
\frac{u_{2}}{J_{x x}} \\
\frac{u 3}{J_{y y}} \\
\frac{u 4}{J_{z z}}
\end{array}\right]
$$

where $\left[\begin{array}{lll}\phi & \theta & \psi\end{array}\right]^{T}$ are Euler angles and known as roll (rotation around $x$-axis), pitch (rotation around $y$-axis) and yaw (rotation around $z$-axis). $\boldsymbol{J}$ is an inertia matrix $(\boldsymbol{J}=$ $\left.\operatorname{diag}\left[J_{x x}, J_{y y}, J_{z z}\right]\right)$ of the quadrotor and $\left[\begin{array}{lll}u_{2} & u_{3} & u_{4}\end{array}\right]^{T}$ presents the total moments acting on the quadrotor in the body frame. $J_{r}$ denotes the propellers' inertia and $\Omega_{r}$ represents the relative propeller's speed.

\section{Sliding Mode Control Design}

The quadrotor UAV model developed in the previous section can be reformulated as a sixth-order nonlinear dynamical system:

$$
\begin{aligned}
& \dot{x}_{1}=x_{2} \\
& \dot{x}_{2}=F\left(x_{1}, x_{2}\right)+G\left(x_{1}, x_{2}\right) u+D\left(x_{1}, x_{2}, t\right)
\end{aligned}
$$

where $\boldsymbol{x} \in \mathbb{R}^{6}$ represents the system state vector as $\boldsymbol{x}=\left[\begin{array}{ll}\boldsymbol{x}_{\mathbf{1}} & \boldsymbol{x}_{\mathbf{2}}\end{array}\right]^{T}$ in which $\boldsymbol{x}_{\mathbf{1}}=\left[\begin{array}{lll}\phi & \theta & \psi\end{array}\right]^{T}$ and $\boldsymbol{x}_{\mathbf{2}}=\left[\begin{array}{lll}\dot{\phi} & \dot{\theta} & \dot{\psi}\end{array}\right]^{T} \cdot \boldsymbol{F}\left(\boldsymbol{x}_{1}, \boldsymbol{x}_{2}\right)=\left[\begin{array}{lll}F_{1} & F_{2} & F_{3}\end{array}\right]^{T}$ and $\boldsymbol{G}\left(\boldsymbol{x}_{1}, \boldsymbol{x}_{2}\right)=\left[\begin{array}{lll}G_{1} & G_{2} & G_{3}\end{array}\right]^{T}$ are two nonlinear functions describing system dynamics, and $\boldsymbol{u} \in \mathbb{R}^{3}$ is the control input to be designed. $D\left(x_{1}, x_{2}, t\right)$ is the time-varying external disturbance or parametric uncertainties acting on the 
UAV dynamic model. In the following, the design methodology of the proposed technique is compared with conventional sliding mode control (CSMC).

\section{A. Conventional Sliding Mode Control (CSMC)}

A conventional sliding manifold can be expressed as:

$$
\sigma_{C S M C}=\tilde{x}_{2}+\Lambda \tilde{x}_{1}
$$

where $\sigma_{C S M C}=\left[\begin{array}{lll}\sigma_{1, C S M C} & \sigma_{2, C S M C} & \sigma_{3, C S M C}\end{array}\right]^{T}$, denotes a conventional sliding surface vector, $\tilde{\boldsymbol{x}}_{1}=\boldsymbol{x}_{1}-\boldsymbol{x}_{1 d}$ and $\tilde{x}_{2}=x_{2}-x_{2 d}$ are perturbations from desired points $\left(\boldsymbol{x}_{1 \boldsymbol{d}}=\left[\begin{array}{lll}\phi_{d} & \theta_{d} & \psi_{d}\end{array}\right]^{T}, \boldsymbol{x}_{\mathbf{2 d}}=\left[\begin{array}{lll}\dot{\phi}_{d} & \dot{\theta}_{d} & \dot{\psi}_{d}\end{array}\right]^{T}\right) \cdot \boldsymbol{\Lambda}=$ $\operatorname{diag}\left[\lambda_{1}, \lambda_{2}, \lambda_{3}\right]$ contains positive constants. These constants $\left(\lambda_{i} ; i=1,2,3\right)$ are typically limited by three factors [19]: the frequency of the lowest unmodeled structural resonant mode $\left(\nu_{r}\right)$, the largest neglected time delay $\left(T_{d}\right)$, and the sampling rate $\left(\nu_{s}\right)$ as follows:

$$
\lambda_{i} \leq \frac{2 \pi}{3} \nu_{r} \quad ; \quad \lambda_{i} \leq \frac{1}{3 T_{d}} \quad ; \quad \lambda_{i} \leq \frac{1}{5} \nu_{s}
$$

To ensure the attractiveness of the sliding manifold, the following positive definite function of $\sigma_{C S M C}$ can be considered as a Lyapunov function

$$
V=\frac{1}{2} \sigma_{C S M C}^{T} \sigma_{C S M C}
$$

Taking the derivative of Eq. (5) with respect to time results in

$$
\begin{aligned}
\dot{V}= & \left(\sigma_{1, C S M C} \dot{\sigma}_{1, C S M C}\right)+\left(\sigma_{2, C S M C} \dot{\sigma}_{2, C S M C}\right)+ \\
& \left(\sigma_{3, C S M C} \dot{\sigma}_{3, C S M C}\right)
\end{aligned}
$$

According to the Lyapunov's direct method, the negative definiteness of $\dot{V}$ implies that the equilibrium state at the origin is stable i.e.,

$$
\sigma_{i, C S M C} \dot{\sigma}_{i, C S M C}=-\mu_{i}\left|\sigma_{i, C S M C}\right| ; i=1,2,3
$$

where the parameter $\mu_{i}$ is a positive constant which must be greater than the magnitude of the disturbance. Substituting Eq. (2) into Eq. (7) results in

$$
F_{i}+G_{i} u_{i+1}-\dot{x}_{2 d, i}+\lambda_{i} \tilde{x}_{2, i}=-\mu_{i} \operatorname{sign}\left(\sigma_{i, C S M C}\right)
$$

Therefore, for the nonlinear dynamical system in Eq. (2) with the conventional sliding manifold defined in Eq. (3) the stablizing controller can be designed as

$$
u_{i+1}=-G_{i}{ }^{-1}\left[\mu_{i} \operatorname{sign}\left(\sigma_{i, C S M C}\right)+\lambda_{i} \tilde{x}_{2, i}+F_{i}-\dot{x}_{2 d, i}\right]
$$

then the system's states move toward the sliding surface and the motion is confined to it.

According to Eq. (9), $\operatorname{sign}\left(\sigma_{C S M C}\right)$ is a signum function. Signum function is a discontinuous function that can switch the control signal at an infinite frequency, and thereby excite the unmodeled fast dynamics or undesired oscillations known as chattering. To solve this problem simply, two techniques can be employed. In the first technique, one may approximate the discontinuous function with a hyperbolic switching function known as Boundary Layer (BL) as:

$$
u_{i+1}=-G_{i}^{-1}\left[\mu_{i} \tanh \left(\sigma_{i, C S M C}\right)+\lambda_{i} \tilde{x}_{2, i}+F_{i}-\dot{x}_{2 d, i}\right]
$$

or Continuous SMC technique (Cnt.) [17]

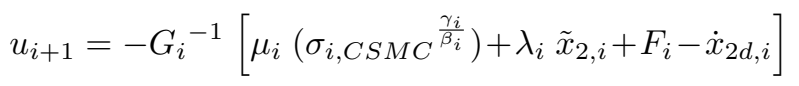

where $\gamma_{i}$ and $\beta_{i}$ are positive odd integers. Though the continuous approximation may not guarantee the robust performance of the system in the boundary layer, continuous SMC approach can be utilized. Sliding mode control techniques using boundary layers are still considered as a promising method to alleviate the chattering phenomenon [20].

\section{B. Intercept-Varying Sliding Mode Control (IVSMC)}

To enhance the performance of CSMC, in this subsection two novel continuous time-varying switching surfaces are introduced. Since system's trajectory is highly sensitive to external disturbances or parameter variations in the reaching phase, one of the methods to increase the robust performance of the SMC technique is to eliminate the reaching phase via shifting sliding manifolds. Figure 2 shows a procedure whereby the proposed sliding surface moves over time based on initial conditions and converges to the desired sliding manifold. This method suggests that the intercept-varying sliding surface $\left(\sigma_{I V}\right)$ can be selected in the whole phase plane either $\left.\tilde{x} \dot{\tilde{x}}\right|_{t=0} \geq 0$ or $\left.\tilde{x} \dot{\tilde{x}}\right|_{t=0}<0$. Main features of the proposed intercept-varying sliding mode control (IVSMC) approaches are

- Simplicity of the proposed approaches.

- The proposed time-varying functions can be combined with various SMC algorithms such as terminal SMC, nonsingular terminal SMC, higher-order SMC, twisting and super-twisting algorithms.

- The proposed approaches show a robust performance for all time instances of the reaching phase further to the sliding manifold.

- It is not necessary to determine switching parameters using time-consuming and complicated algorithms. The proposed approach is able to determine its parameters quickly and easily.

1) First Proposed Approach (IVSMC1): In this approach, intercept-varying sliding manifolds are selected such that the time required from initial sliding surface to the desired one is the same with the reaching time in CSMC. Desirably, the following sliding surface is proposed

$$
\sigma_{I V}=\tilde{x}_{2}+\Lambda \tilde{x}_{1}+f(t)
$$

where $\sigma_{I V}$ is a proposed sliding surface, $f(t)$ is a vector including nonlinear time-varying functions as $f(t)=$ $\left[\begin{array}{lll}f_{1}(t) & f_{2}(t) & f_{3}(t)\end{array}\right]^{T}$.

The design procedure of a nonlinear vector, $f(t)$, is introduced in the following. The initial value of $f(t)$ must be 


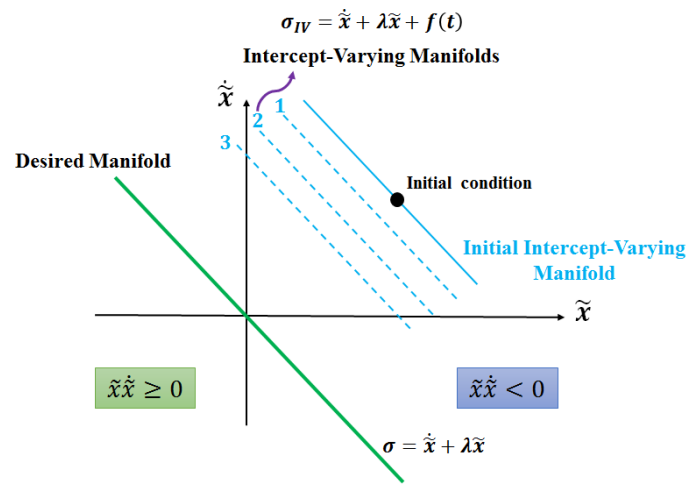

Fig. 2. Intercept-varying sliding manifolds based on initial conditions

defined such that the initial states lie on the sliding manifold. i.e.,

$$
\sigma_{I V}(0)=0 \Rightarrow f(0)=-\left(\tilde{x}_{2}(0)+\Lambda \tilde{x}_{1}(0)\right)
$$

Then, the nonlinear vector $f(t)$ must be chosen such that the intercept-varying sliding manifold reaches the desired manifold. Here, the following nonlinear function is proposed

$$
f_{i}(t)=f_{i}(0)\left(1-\tanh \alpha_{i} t\right) ; i=1,2,3
$$

where $\alpha_{i}$ is a positive constant and will be determined later. As a result, the proposed intercept-varying sliding mode controller, $u_{i+1, I V}$ is derived as

$$
\begin{gathered}
u_{i+1, I V}=-G_{i}{ }^{-1}\left[\mu_{i} \operatorname{sign}\left(\sigma_{i, I V}\right)+\lambda_{i} \tilde{x}_{2, i}+F_{i}-\dot{x}_{2 d, i}-\right. \\
\left.f_{i}(0) \alpha_{i}\left(1-\tanh ^{2} \alpha_{i} t\right)\right]
\end{gathered}
$$

Reaching time in CSMC can be estimated using

$$
t_{r, i}=\frac{\left|\sigma_{i, C S M C}(0)\right|}{\mu_{i}}
$$

the minimum value of $\alpha t_{r}$ must be computed such that $\tanh \alpha t_{r}=1$. This implies that the proposed approach guarantees the finite reaching time to the desired sliding manifold. In MATLAB, $\tanh 5.3=1$ can be achieved if the error tolerance is set to $10^{-4}$. As a consequence, the magnitude of the parameter $\alpha_{i}$ can be obtained using the following relation:

$$
\alpha_{i}=\frac{5.3 \mu_{i}}{\left|\sigma_{i, C S M C}(0)\right|}
$$

Eq. (17) clearly shows the applicability of the designed parameter $\alpha_{i}$ in a continuous time.
2) Second Proposed Approach (IVSMC2 and IVSMC3): In the second approach, the time required from initial sliding surface to the desired one can be selected by a designer optionally. On the other hand, one can accelerate or decelerate the reaching phase in CSMC. Desirably, the following sliding surface is proposed

$$
\sigma_{I V}=\tilde{x}_{2}+\Lambda \tilde{x}_{1}+h(t)
$$

where $\boldsymbol{h}(\boldsymbol{t})$ is a vector including time-varying functions as $\boldsymbol{h}(\boldsymbol{t})=\left[\begin{array}{lll}h_{1}(t) & h_{2}(t) & h_{3}(t)\end{array}\right]^{T}$. To accelerate or decelerate the reaching phase, $\boldsymbol{h}(\boldsymbol{t})$ can be selected as

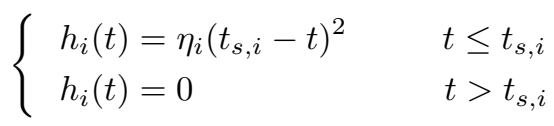

where $\eta_{i}$ is a positive constant and $t_{s, i}$ can be selected by a designer. In this paper, $t_{s, 1}=0.3 \mathrm{~s}$ and $t_{s, 2}=4 \mathrm{~s}$ are considered as IVSMC2 and IVSMC3, respectively. Subsequently, the proposed intercept-varying sliding mode controller, $u_{i+1, I V}$ can be derived as

$$
\begin{gathered}
u_{i+1, I V}=-G_{i}^{-1}\left[\mu_{i} \operatorname{sign}\left(\sigma_{i, I V}\right)+\lambda_{i} \tilde{x}_{2, i}+F_{i}-\dot{x}_{2 d, i}-\right. \\
\left.2 \eta_{i}\left(t_{s, i}-t\right)\right]
\end{gathered}
$$

Eq. (20) can be used when $t \leq t_{s, i}$. Similarly, the derived controller in Eq. (10) can be used when $t>t_{s, i}$.

\section{RESUlTS AND DISCUSSION}

This section is dedicated to simulation of the time-varying sliding mode control approaches for stabilizing the attitude of a quadrotor UAV. Simulations are developed utilizing the MATLAB software. Physical parameters of an AR Drone Parrot 2.0 are given in Table I.

To investigate the effectiveness of the proposed controllers, the following mission subject to disturbances as $D(t)=$ $10^{-2}\left[\begin{array}{lll}4 \sin (t) & 3 \sin (t) & 2 \cos (t)\end{array}\right]^{T}$ is addressed. The mission task is to set Euler angles from initial conditions as $\left[\begin{array}{lll}\phi(0) & \theta(0) & \psi(0)\end{array}\right]=\left[\begin{array}{lll}60 & 0 & 0\end{array}\right]$ deg to desired conditions as $\left[\begin{array}{lll}\phi_{d} & \theta_{d} & \psi_{d}\end{array}\right]=\left[\begin{array}{lll}30 & 45 & -60\end{array}\right]$ deg. Initial and desired conditions for Euler rates are both selected as $\left[\begin{array}{lll}\dot{\phi}(0) & \dot{\theta}(0) & \dot{\psi}(0)\end{array}\right]=\left[\begin{array}{ccc}0 & 0 & 0\end{array}\right] \mathrm{rad} / \mathrm{s}$. The uncertainty in a first component of the inertia matrix of the quadrotor is also applied for this mission as $J_{x x}{ }^{\prime}=7.2 \times 10^{-3}$. Sliding gains are selected as $\lambda_{1}=\lambda_{2}=\lambda_{3}=1$ and $\mu_{1}=\mu_{2}=\mu_{3}=$

TABLE I

Physical Parameters of THE QuAdrotor Model

\begin{tabular}{|c|c|}
\hline Symbol & Value \\
\hline$J_{x x}=J_{y y}$ & $1.8 \times 10^{-3}\left(\mathrm{~kg} \mathrm{~m}^{2}\right)$ \\
\hline$J_{z z}$ & $4.7 \times 10^{-3}\left(\mathrm{~kg} \mathrm{~m}^{2}\right)$ \\
\hline$J_{r}$ & $1.8 \times 10^{-5}\left(\mathrm{~kg} \mathrm{~m}^{2}\right)$ \\
\hline
\end{tabular}




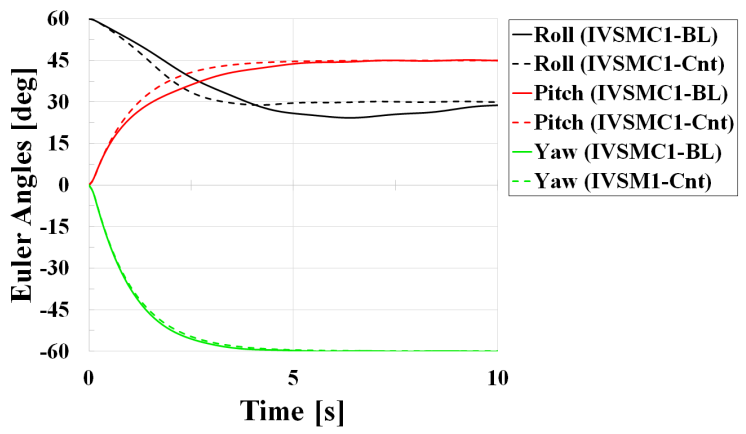

Fig. 3. Time history of Euler angles based on IVSMC1-BL and IVSMC1-Cnt subject to uncertainty and external disturbances

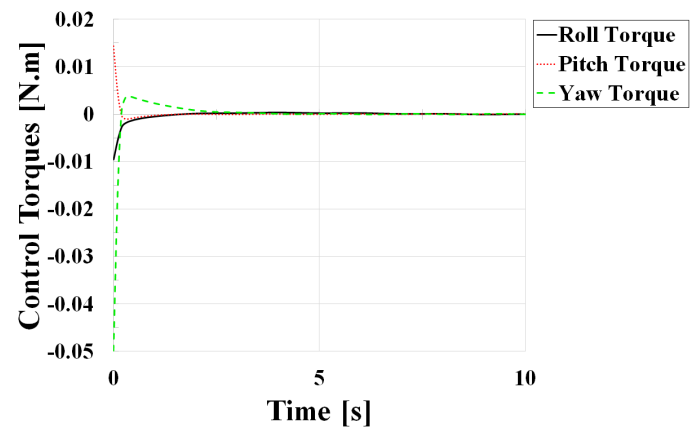

Fig. 4. Time history of control torques based on IVSMC1-BL subject to uncertainty and external disturbances

1. Fractional exponents of the continuous SMC are assumed as $\gamma_{1}=\gamma_{2}=\gamma_{3}=7$ and $\beta_{1}=\beta_{2}=\beta_{3}=17$.

Attitude tracking of the quadrotor subject to disturbances and uncertainty based on the IVSMC1-BL and IVSMC1-Cnt methods is shown in Fig. 3 using the first approach. Fig. 3 shows that Euler angles start from their initial conditions and converge to the predetermined desired values. Furthermore, as it can be clearly seen from Fig. 3, the perturbation of the roll angle around the desired condition in IVSMC1-BL is higher than that in IVSMC1-Cnt. Time responses of control torques are illustrated in Figs. 4 and 5 based on IVSMC1-BL and IVSMC1-Cnt, respectively. Fig. 5 displays approximately additional control effort in the roll channel to justify the roll angle. Time history of Euler angles based on IVSMC2-Cnt and IVSMC3-Cnt is shown in Fig. 6 for two assumed reaching time as $t_{s 1}=0.3 \mathrm{~s}$ and $t_{s 2}=4 \mathrm{~s}$. As expected, the second proposed approach could successfully accelerate or decelerate the reaching phase in the sliding mode control theory.

Time responses of Euler angles deviation against disturbances and the inertia uncertainty are represented in Figs. 7 and 8 based on the IVSMC1-BL, and three proposed controllers as IVSMC1-Cnt, IVSMC2-Cnt and IVSMC3-Cnt approaches. The comparison between IVSMC-BL and IVSMCCnt in Fig. 7 proves that the IVSMC-Cnt has better robust performance rather than IVSMC-BL in the presence of environmental disturbances and parameter uncertainties.

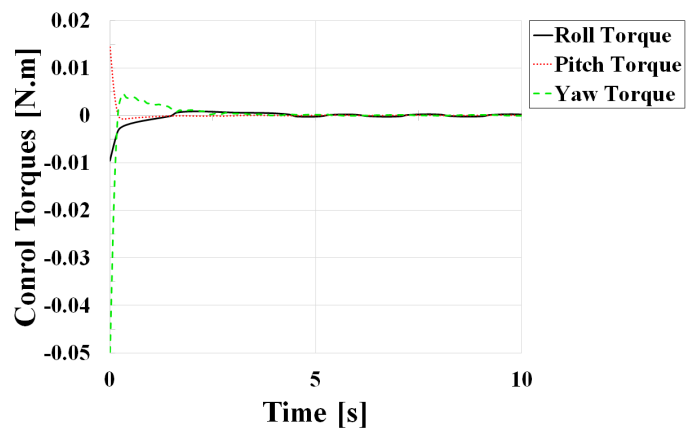

Fig. 5. Time history of control torques based on IVSMC1-Cnt subject to uncertainty and external disturbances

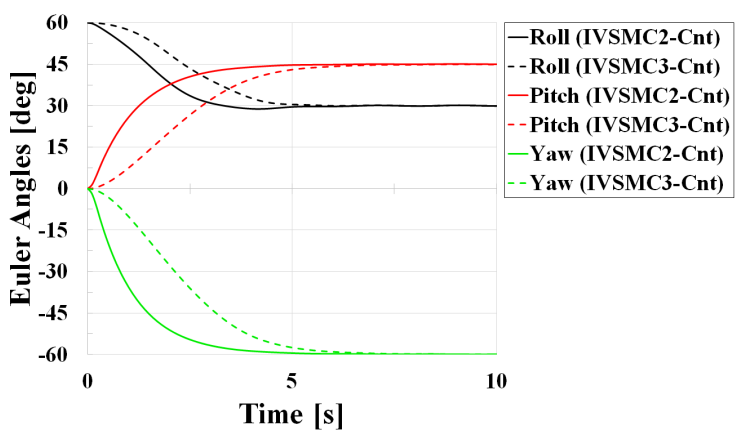

Fig. 6. Time history of Euler angles based on IVSMC2-Cnt and IVSMC3-Cnt subject to uncertainty and external disturbances

Generally, boundary layer technique has a major drawback not to guarantee the robust performance of the system in the boundary layer thickness. Table II displays the root-meansquare $(\mathrm{rms})$ value of roll torque $\left(u_{2}\right)$ and roll error $\left(\tilde{x}_{1}\right)$, as an example, based on the proposed control approaches in the presence of external disturbances and inertia uncertainty. The effectiveness of the IVSMC-Cnt in robust performance and disturbance rejection is represented in Table II. Fig. 8 represents that the perturbation of Euler angles from desired values based on the IVSMC3-Cnt is lower than that based on the IVSMC2-Cnt in the transient phase. However, Table II presents that the perturbation of Euler angles from desired values based on the IVSMC3-Cnt is higher than that based on the IVSMC2-Cnt in the steady-state phase.

TABLE II

Root-Mean-Square Values of THE Proposed Controllers

\begin{tabular}{|c|c|c|}
\hline $\begin{array}{c}\text { Proposed } \\
\text { Approaches }\end{array}$ & $\begin{array}{c}\text { Roll error } \\
\text { (deg) }\end{array}$ & $\begin{array}{c}\text { Roll torque } \\
\text { (N.m) }\end{array}$ \\
\hline IVSMC1-BL & 11.2671 & $1.1829 \times 10^{-3}$ \\
\hline IVSMC1-Cnt & 9.7624 & $1.3069 \times 10^{-3}$ \\
\hline IVSMC2-BL & 11.3950 & $9.6995 \times 10^{-4}$ \\
\hline IVSMC2-Cnt & 9.8866 & $1.1203 \times 10^{-3}$ \\
\hline IVSMC3-BL & 14.9292 & $3.3585 \times 10^{-4}$ \\
\hline IVSMC3-Cnt & 12.9174 & $4.5614 \times 10^{-4}$ \\
\hline
\end{tabular}




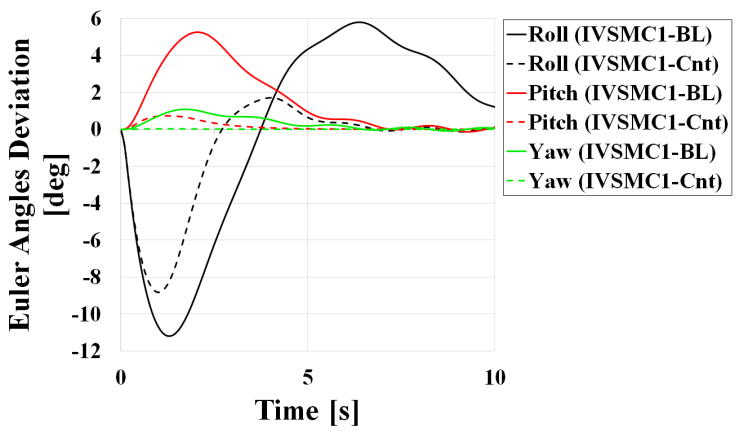

Fig. 7. Time history of Euler angles deviation based on IVSMC1-BL and IVSMC1-Cnt subject to uncertainty and external disturbances

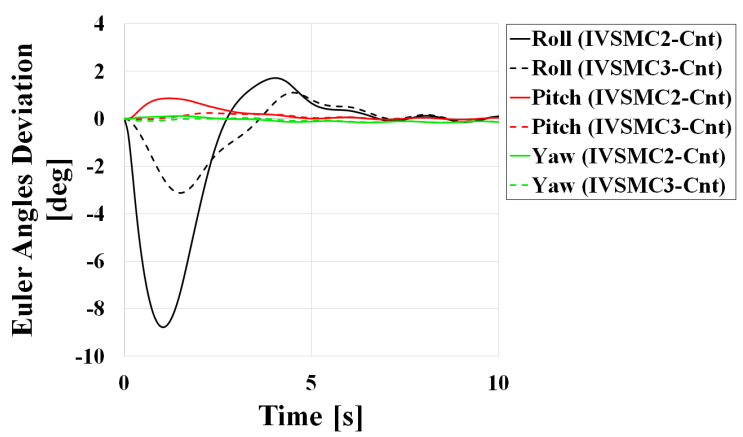

Fig. 8. Time history of Euler angles deviation based on IVSMC2-Cnt and IVSMC3-Cnt subject to uncertainty and external disturbances

\section{CONCLUSIONS}

In this paper, an effective implementation of two novel approaches for stabilizing the attitude of a quadrotor UAV have been developed based on the concept of time-varying sliding mode control. Since conventional sliding mode controllers are sensitive against environmental disturbances and parameter uncertainties in the reaching phase, two new intercept-varying sliding mode control (IVSMC) strategies are proposed via shifting the initial sliding manifold to improve the robust performance of the system subject to external disturbances and uncertainties. The proposed sliding mode control methodologies are easy and they can be applied to various types of linear/nonlinear sliding surfaces. Furthermore, they can retain the robust performance of the designed controllers continuously in time. Moreover, to eliminate the chattering drawback, two methods have been employed as boundary layer (known as IVSMC-BL) and continuous sliding mode control (known as IVSMC-Cnt). Finally, the proposed IVSMC approaches are applied to a quadrotor UAV in the presence of external disturbances and the inertia uncertainty. The obtained results proves that the IVSMC-Cnt has better robust performance than IVSMC-BL against parameter uncertainties and environmental disturbances. In addition, the reaching phase could be accelerated or decelerated using the proposed timevarying manifolds.

\section{ACKNOWLEDGMENT}

The authors are grateful for the support of the National Nuclear Laboratory (NNL) and the Nuclear Decommissioning Authority (NDA).

\section{REFERENCES}

[1] T. Robinson, "Challenges for Robotics in Nuclear Decommissioning," E\&T Nuclear Energy, August 2017. (https://energyhub.theiet.org/users/59438-tom-robinson/)

[2] A. Montazeri, and J. U. Ekotuyo, "Development of dynamic model of a 7DOF hydraulically actuated tele-operated robot for decommissioning applications," IEEE American Control Conference (ACC), 2016, pp. 1209-1214.

[3] A. Montazeri, C. West, S. D. Monk, and C. J. Taylor, "Dynamic modeling and parameter estimation of a hydraulic robot manipulator using a multi-objective genetic algorithm," International Journal of Control, vol. 90, no. 4, 2017, pp. 661-683.

[4] A. Montazeri, and J. Poshtan, "Optimizing a Multi-Channel ANC System for Broadband Noise Cancellation in a Telephone Kiosk Using Genetic Algorithms," Shock and Vibration, vol. 16, no. 3, 2009, pp. 241-260.

[5] A. Montazeri, and J. Poshtan, "GA-based optimization of a MIMO ANC system considering coupling of secondary sources in a telephone kiosk", Applied Acoustics, vol. 70, no. 7, 2009, pp. 945-953.

[6] J.-J. Xiong, and E.-H. Zheng, "Position and attitude tracking control for a quadrotor UAV," ISA Transactions, vol. 53, pp. 725-731, May 2014.

[7] B. Abci, G. Zheng, D. Efimov, and M. E. B. E. Najjar, "Robust altitude and attitude sliding mode controllers for quadrotors," IFACPapersOnLine, vol. 50, pp. 2720-2725, July 2017.

[8] U. Ansari, and A. H. Bajodah, "Robust generalized dynamic inversion quadrotor control," IFAC-PapersOnLine, vol. 50, pp. 8181-8188, July 2017.

[9] Z. Jia, J. Yu, Y. Mei, Y. Chen, Y. Shen, and X. Ai, "Integral backstepping sliding mode control for quadrotor helicopter under external uncertain disturbances," Aerospace Science and Technology, vol. 68, pp. 299-307, September 2017.

[10] G. P. Matthews, and R. A. DeCarlo, "Decentralized tracking for a class of interconnected nonlinear systems using variable structure control," Automatica, vol. 24, no. 2, pp. 817-193, 1988.

[11] M. Rubagotti, A. Estrada, F. Castanos, A. Ferrara, and L. Fridman, "Integral sliding mode control for nonlinear systems with matched and unmatched perturbations," IEEE Transactions on Automatic Control, vol. 56, no. 11, pp. 2699-2704, 2011.

[12] S.-C. Tan, Y. M. Lai, and C. K. Tse, "Indirect sliding mode control of power converters via double integral sliding surface," IEEE Transactions on Power Electronics, vol. 23, no. 2, pp. 600-611, 2008.

[13] M. Asif, M. J. Khan, and N. Cai, "Adaptive sliding mode dynamic controller with integrator in the loop for nonholonomic wheeled mobile robot trajectory tracking," International Journal of Control, vol. 87, no. 5, pp. 964-975, 2014.

[14] S.-B. Choi, D.-W. Park, and S. Jayasuriya, "A time-varying sliding surface for fast and robust tracking control of second-order uncertain systems," Automatica, vol. 30, pp. 899-904, 1994.

[15] A. Bartoszewicz, "A comment on a time-varying sliding surface for fast and robust tracking control of second-order uncertain systems," Automatica, vol. 31, pp. 1899-1895, 1995.

[16] J. G. Y. Sheng, and X. Liu, "Second-order time-varying sliding mode control for reentry vehicle," International Journal of Intelligent Computing and Cybernetics, vol. 6, pp. 272-295, 2013.

[17] H. Nemati, M. Oishi, N. Kobayashi, R. Nakata, and S. Hokamoto, "New continuous sliding mode design for spacecraft attitude control," $24^{\text {th }}$ Workshop on JAXA Astrodyn. Flight Mech., Japan, July 2014.

[18] H. Nemati, A. Naghash, S. Mozafari, and A. Jamei, "Robust attitude control for quadrotors with external disturbances," $9^{\text {th }}$ International Micro Air Vehicles Conference and Flight Competition (IMAV), France, pp. 81-88, September 2017.

[19] J.-J. E. Slotine, and W. P. Li, Applied Nonlinear Control, New Jersey: Prentice Hall, 1991.

[20] B. Yang, T. Yu, H. Shu, J. Dong, and L. Jiang, "Robust sliding-mode control of wind energy conversion systems for optimal power extraction via nonlinear perturbation observers," Applied Energy, vol. 210, pp. 711$723,2018$. 